\title{
SIMULTANEOUS SPECTROPHOTOMETRIC QUANTITATION OF RUTIN AND CHLOROGENIC ACID IN LEAVES OF Ribes uva-crispa L. BY ONE-DIMENSIONAL CONTINUOUS WAVELET TRANSFORMS
}

\author{
GÜLSEN KENDIR ${ }^{a}, A Y S ̧ E G \ddot{U} L K \ddot{O R O G} L U^{b, c} A N D$ ERDAL DINÇ ${ }^{d^{*}}$ \\ ${ }^{a}$ Süleyman Demirel University, Faculty of Pharmacy, Department of Pharmaceutical Botany, 32260 Isparta-Turkey. \\ ${ }^{b}$ Ankara University Faculty of Pharmacy, Department of Pharmaceutical Botany, 06100 Tandoğan, Ankara-Turkey. \\ 'Afyonkarahisar Health Sciences University, Faculty of Pharmacy, Department of Pharmaceutical Botany, 03200 Afyon, Turkey. \\ 'Ankara University Faculty of Pharmacy, Department of Analytical Chemistry, 06100 Tandoğan, Ankara-Turkey.
}

\begin{abstract}
New signal processing methods based on one-dimensional continuous wavelet transforms were proposed for the simultaneous quantification of chlorogenic acid and rutin in the leaves of Ribes uva-crispa L. These methods are based on the application of continuous wavelet transforms to the absorption spectral bands of the analyzed compounds and their samples. In preliminary practices, symlets 8 and biorthogonal 4.4 continuous wavelet transforms (sym8-CWT and bior4.4-CWT) among several wavelet families were found to be optimal ones to perform a selective and sensitive chemical determination of the analytes in analyzed plant samples. Linear regression lines were obtained by measuring sym8-CWT and bior4.4-CWT amplitudes at 333.5 and $336.0 \mathrm{~nm}$ for chlorogenic acid and 351.5 and $350.0 \mathrm{~nm}$ for rutin in the spectral region $250-450 \mathrm{~nm}$. Linear regression curves were found to be linear in the working range of $2.5-40.0 \mu \mathrm{g} / \mathrm{mL}$ for both compounds. The validation and analytical applicability of the proposed sym8-CWT and bior4.4-CWT approaches were confirmed by analyzing the synthetics mixtures and standard addition samples. It was observed that the application of both sym8-CWT and bior4.4-CWT tools is very appropriate for the simultaneous spectral quantification of chlorogenic acid and rutin in the real plant samples consisting of leaves of $R$. uva-crispa.
\end{abstract}

Keywords: Continuous wavelet transforms; Simultaneous spectrophotometric quantitation; Chlorogenic acid, Rutin, Leaves of Ribes uva-crispa L.

\section{INTRODUCTION}

Nowadays, high-performance liquid chromatography (HPLC) or ultraperformance liquid chromatography (UPLC) is one of the most popular and useful procedures used in analytical chemistry for the analysis of active compounds from plant samples, pharmaceutical and food products. However, in many cases, HPLC (or UPLC) may not give the expected analysis results of valuable chemicals from plants samples due to experimental factors, such as sample matrix effect, improper column and mobile phase usage. In addition, Chromatographic separation of analytes may be resulted in the co-elution of peaks of analytes in a chromatogram due to very similar physicochemical characteristics of analytes. In order to perform the analysis of complex samples, such as extracts obtained from plants, with a chromatographic method, it may be required long preliminary experiments and tedious studies for searching optimal chromatographic separation conditions causing high cost. In this context, derivative spectrophotometry instead of HPLC method is an alternative method, particularly for the quantitative resolution of a two-component mixture. In some cases, the derivative spectrophotometry may not provide a quantitative resolution of binary mixture systems due to complexity of samples. Derivative spectrophotometry and some applications were reported in the literature [1-2]. To overcome the above mentioned drawbacks of the analysis with HPLC and derivative spectrophotometry, researchers need to develop new, more selective, sensitive, accurate, precise, and easy applicable analytical methods for the efficient resolution of the analytical problems with low cost.

To increase the potential power of the spectrophotometric analysis, one of the newest signal processing tools is wavelets. Wavelet transform (WT) has become an important tool for data reduction, de-noising, baseline correction, and resolution of overlapping spectra of analytes in complex mixtures with many applications e.g. analysis of pharmaceuticals or the analysis of analytes [3-5]. WT is classified into discrete wavelet and continuous wavelet analysis [6]. In recent studies, the combined use of the CWT methods with zero-crossing technique and ratio signals has opened a new gate for the simultaneous spectrophotometric resolution of binary and ternary mixtures without needing any separation step [7-16].

Ribes uva-crispa $\mathrm{L}$. is spiny shrubs, 1-1.5 $\mathrm{m}$ long with 2-3 spines at the nodes. Its leaves are simple, palmately lobed, scarcely pubescent or glabrous, truncate or subcordate at the base. The flowers are pale green, sometimes pinkish in axillary clusters of 1-3. The fruits are globose to ovoid, green, yellow or purplishred [17]. The fruits of $R$. uva-crispa contain flavonoids, tannins, anthocyanins, phenolic acids, and lipids (18-24). Chemical and biological activities studies on R. uva-crispa leaves are limited. The presence of cyanogenic glucosides (e.g. hydroxynitrile glucosides), proanthocyanidin (e.g. prodelphinidin and procyanidin), flavonoid (e.g. rutin, myricetin, quercetin, kaempferol), and phenolic acid (e.g. chlorogenic acid) in $R$. uva-crispa leaves were demonstrated (25-27). Hypotensive, antimicrobial, antioxidant, and cytotoxic effects of the leaves were uncovered (28-31). Fruits of $R$. uva-crispa are employed as purgative, diuretic, stomachic in the folk medicine of Turkey (32). R. uva-crispa leaves are utilized as an astringent to treat dysentery and wounds and they are used against gravel (33). Besides, their leaves have been used in the treatment of tuberculosis in Uganda (34).

A literature survey revealed that several analytical methods including HPLC [35], UPLC [27], GC [36], HPTLC [37], HPLC-MS [38-41], UPLC-MS [42], capillary electrophoresis [43-45], voltammetry [46] were used for the analysis of chlorogenic acid and rutin alone or both in plants or samples containing other active compounds. However, there was no study on the simultaneous quantification of the related substances applying continuous wavelet transforms and first derivative spectrophotometry (DS ${ }^{1}$ ) to UV spectra of plant samples.

In this study, the wavelet signal processing tools, the sym8-CWT, and bior4.4CWT were proposed for the simultaneous spectrophotometric determination of chlorogenic acid (CA) and rutin (RUT) in leaves of Ribes uva-crispa. After 
preliminary signal processing tests, sym8 and bior4.4 families were found to be optimal ones to reach better quantification of analytes with the highest recovery results. For comparison, first derivative spectrophotometry was used for the quantification of CA and RUT in the related plant samples. All of the proposed wavelet transform and $\mathrm{DS}^{1}$ methods were validated by analyzing various CARUT mixtures and by using the standard addition technique. Mean recovery results for CA and RUT were obtained as $99.4 \%$ and $98.8 \%$ using sym8-CWT, $99.5 \%$ and $102.7 \%$ using bior4.4-CWT and $101.2 \%$ and $99.1 \%$ using DS $^{1}$, respectively.

In the application of the methods to real plant samples, the successful quantitation results for both compounds were obtained. The analysis results provided by applying the sym8-CWT, bior4.4-CWT and DS ${ }^{1}$ were statistically compared with those obtained by the UPLC method in the literature [27]. It was concluded that the signal-processing methods were very useful and promising techniques for the quantitative analysis of CA and RUT in leaves samples of R. uva-crispa.

\section{EXPERIMENTAL}

\subsection{Instruments and Software}

Spectrophotometric recordings and measurements were performed on a Shimadzu UV-2555 double beam UV-VIS spectrophotometer connected with a computer loaded with Shimadzu UVProbe software. For applying the CWT approaches, data treatments and figure plots were performed on Wavelet Toolbox in Matlab 7.0 software and special wavelet analysis algorithm written in Matlab software. In addition, regression, quantitation, and statistical analysis were made through the Microsoft EXCEL.

\subsection{Sample Preparation}

In the analysis of CA and RUT in leaves of Ribes uva-crispa, medicinal plant samples were collected from Kizılcahamam region (Ankara, Turkey). 5.0 gr of powdered leaves were utilized for the extraction procedure. The extraction process was done using $100 \mathrm{~mL}$ of methanol during 3 days with a moving maceration technique. The organic extract was evaporated on a rotary vacuum evaporator at $40{ }^{\circ} \mathrm{C}$ to dryness. For the analysis of CA and RUT, $1.0 \mathrm{mg}$ of the extracted plant sample was weighed and dissolved in $5 \mathrm{ml}$ of methanol. This sample solution was diluted with methanol to get the concentration range of the calibration eg. The absorption spectra of the resulting plant solutions were recorded.

\subsection{Standard Solutions}

Stock solutions of CA and RUT were prepared by dissolving $5 \mathrm{mg}$ of each compound in $10 \mathrm{~mL}$ methanol. From the stock solutions of the analyzed substances, the calibration solutions for CA and RUT were separately prepared in the concentration range $2.5-40.0 \mu \mathrm{g} / \mathrm{mL}$. An independent validation set of six different mixture solutions of analytes in the working concentration range was prepared. Standard addition samples were prepared by adding the standard solutions of CA and RUT (at concentration levels of 5.0, 20.0, and $30.0 \mu \mathrm{g} / \mathrm{mL}$ ) to the plant samples. As in the calibration samples, the UV-VIS spectra of validation samples were recorded.

\section{RESULT AND DISCUSSION}

\subsection{Spectral method improvement}

The absorption spectra of CA and RUT were recorded in the spectral region of $250.0-457.4 \mathrm{~nm}$. The same procedure was repeated for the samples of validation and plant samples. Figure 1a shows the absorption spectral bands of CA and RUT in the concentration range between $2.5-40.0 \mu \mathrm{g} / \mathrm{mL}$. As can be seen from this figure, the simultaneous quantitative analysis of the samples containing the related analytes is impossible by the direct absorbance measurements due to strong overlapping absorption bands of the analytes. For this reason, it was focused mainly on new signal processing approaches named CWT signal processing tools that were improved for the rapid, accurate, precise and economic quantitative analysis of the plant samples containing CA and RUT. For a comparison, $\mathrm{DS}^{1}$ was used for solving the same problem. The signal analysis methods and their applications for the analysis of analytes in the relevant plant samples were described below.
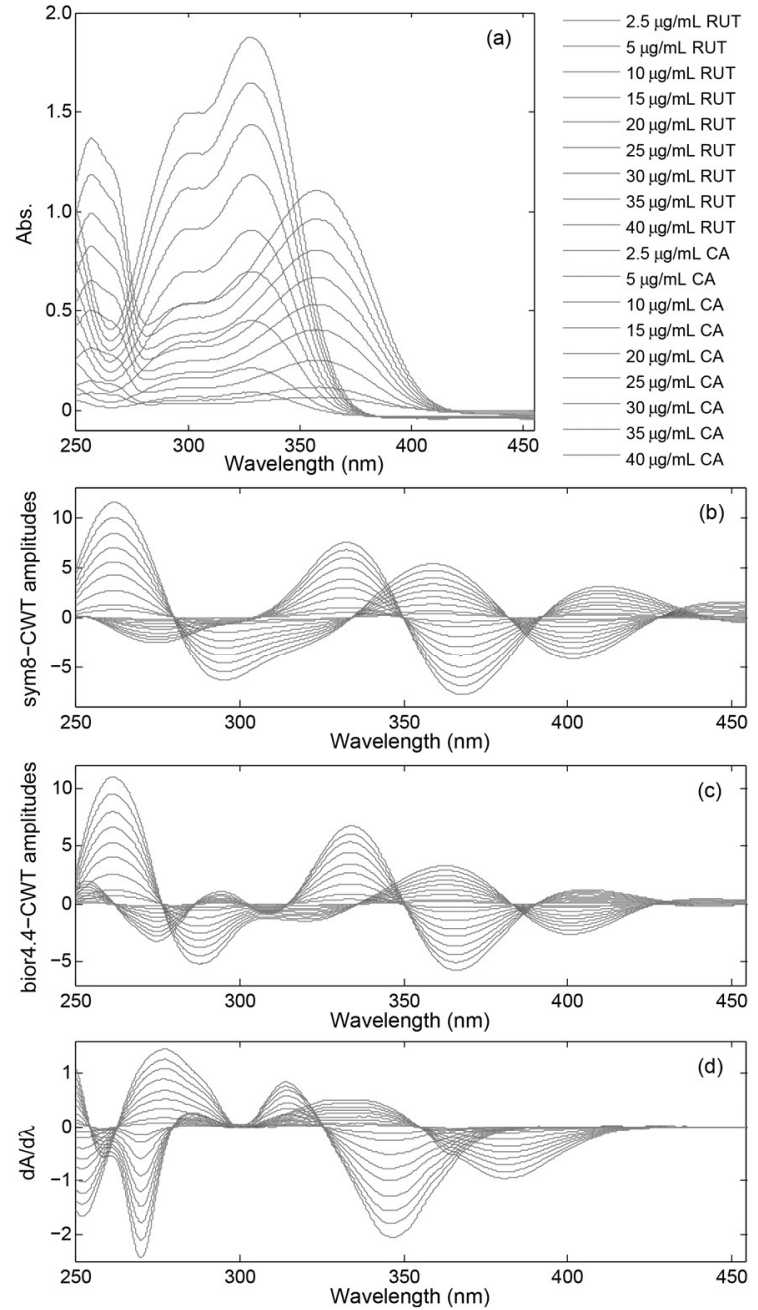

Figure 1. Absorption spectra (a), sym8-CWT spectra (b) and bior4.4-CWT spectra (c) of CA (-) and RUT (-) in the concentration range 2.50-40.00 $\mu \mathrm{g} / \mathrm{mL}$. CA : Chlorogenic acid and RUT: Rutin.

\subsection{Application of the CWT methods}

To find optimal spectral transforms, the application of several wavelet families to the absorption spectra of calibration, validation, and plant samples were tested for their abilities to predict the amount of CA and RUT in the leaves of Ribes uva-crispa. From these signal-processing studies, it was observed that sym8CWT and bior4.4-CWT methods were very suitable for processing the absorption spectra of samples of analytes and plant samples consisting of $R$. $u v a-$ crispa. The sym8-CWT and bior4.4-CWT spectra obtained from the wavelet transforms of the calibration sample samples are presented in Figures $1 \mathrm{~b}$ and $\mathrm{c}$, respectively. It was reported that the concentration of CA and RUT in their samples and for was proportional to the sym8-CWT and bior4.4-CWT amplitudes at 333.5 and $336.0 \mathrm{~nm}$ for CA, and $351.5 \mathrm{~nm}$ and 350.0 for RUT, respectively (see Figure $1 \mathrm{~b}$ and c). To quantify the analytes, the calibration graphs without standard addition for CA and RUT in the concentration range of $2.5-40.0 \mu \mathrm{g} / \mathrm{mL}$ were obtained by regressing the sym8-CWT and bior4.4-CWT amplitudes on the concentration of analytes, respectively. For the calibration curves, the linear regression analysis and its numerical results were summarized in Table 1. The calibration equations obtained were used for the quantitative estimation of herbal compounds (CA and RUT) in the validation and plant samples. To control the matrix effect on the analysis of the respective compounds, the calibration curves of standard additions for both analytes were obtained by using the mathematical relationship between the concentration and CWT signals (sym8-CWT and bior4.4-CWT) at the specified wavelengths. The statistical results of the standard addition calibration curves were given in Table 1. As in the external calibration curves of the analytes, these calibration curves of standard additions were utilized for the quantitation of CA and RUT in the related plant samples. 
.Table 1. Statistical results of the least square regression analysis without standard addition and with standard addition

\begin{tabular}{|c|c|c|c|c|c|c|c|c|c|c|c|}
\hline & & & \multicolumn{9}{|c|}{ Parameter } \\
\hline & Method & Analyte & $\lambda(\mathbf{n m})$ & $\mathbf{m}$ & $\mathbf{n}$ & $\mathbf{r}$ & SE(m) & SE(n) & SE(r) & LOD & LOQ \\
\hline \multirow{6}{*}{ 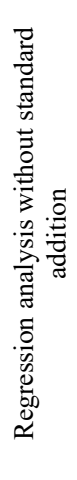 } & \multirow{2}{*}{ sym8-CWT } & $\mathrm{CA}$ & 333.5 & 0.1889 & 0.0422 & 1.0000 & $4.65 \times 10^{-4}$ & $1.11 \times 10^{-2}$ & $1.74 \times 10^{-2}$ & 0.53 & 1.76 \\
\hline & & RUT & 351.5 & 0.1205 & -0.0557 & 0.9999 & $4.01 \times 10^{-4}$ & $1.01 \times 10^{-2}$ & $1.30 \times 10^{-2}$ & 0.76 & 2.52 \\
\hline & \multirow{2}{*}{ bior4.4-CWT } & $\mathrm{CA}$ & 336.0 & 0.1710 & 0.0049 & 0.9997 & $1.62 \times 10^{-3}$ & $3.87 \times 10^{-2}$ & $6.09 \times 10^{-2}$ & 0.68 & 2.26 \\
\hline & & RUT & 350.0 & 0.0541 & -0.0107 & 0.9999 & $1.51 \times 10^{-4}$ & $3.60 \times 10^{-3}$ & $5.66 \times 10^{-3}$ & 0.20 & 0.67 \\
\hline & \multirow{2}{*}{$\mathrm{DS}^{1}$} & $\mathrm{CA}$ & 356.0 & -0.0369 & 0.0210 & 0.9997 & $3.15 \times 10^{-4}$ & $7.50 \times 10^{-3}$ & $1.18 \times 10^{-2}$ & 0.61 & 2.03 \\
\hline & & RUT & 390.0 & -0.0201 & 0.0111 & 0.9994 & $1.84 \times 10^{-4}$ & $4.38 \times 10^{-3}$ & $6.89 \times 10^{-3}$ & 0.65 & 2.18 \\
\hline \multirow{6}{*}{ 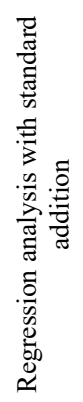 } & \multirow{2}{*}{ sym8-CWT } & CA & 333.5 & 0.1896 & 0.7348 & 1.0000 & $1.32 \times 10^{-3}$ & $2.76 \times 10^{-2}$ & $2.34 \times 10^{-2}$ & 0.44 & 1.46 \\
\hline & & RUT & 351.5 & 0.1173 & 0.1720 & 0.9998 & $1.69 \times 10^{-3}$ & $3.56 \times 10^{-2}$ & $3.01 \times 10^{-2}$ & 0.91 & 3.03 \\
\hline & \multirow{2}{*}{ bior4.4-CWT } & $\mathrm{CA}$ & 336.0 & 0.1692 & -0.0575 & 1.0000 & $1.24 \times 10^{-3}$ & $2.61 \times 10^{-2}$ & $2.21 \times 10^{-2}$ & 0.46 & 1.54 \\
\hline & & RUT & 350.0 & 0.0544 & 0.0673 & 1.0000 & $2.72 \times 10^{-4}$ & $5.73 \times 10^{-3}$ & $4.85 \times 10^{-3}$ & 0.32 & 1.05 \\
\hline & \multirow{2}{*}{$\mathrm{DS}^{1}$} & $\mathrm{CA}$ & 356.0 & -0.0371 & -0.1455 & 0.9998 & $6.81 \times 10^{-4}$ & $1.43 \times 10^{-2}$ & $1.21 \times 10^{-2}$ & 1.16 & 3.86 \\
\hline & & RUT & 390.0 & -0.0202 & -0.0279 & 1.0000 & $1.12 \times 10^{-4}$ & $2.36 \times 10^{-3}$ & $2.00 \times 10^{-3}$ & 0.35 & 1.16 \\
\hline
\end{tabular}

SE (m) : Standard error of intercept

SE (n) : Standard error of slope

SE (r) : Standard error of correlation coefficient

LOD : Limit of detection $(\mu \mathrm{g} / \mathrm{mL})$

LOQ : Limit of quantitation $(\mu \mathrm{g} / \mathrm{mL})$

\subsection{Application of the $\mathrm{DS}^{1}$ method}

To compare the analysis results obtained by applying the sym8-CWT and bior4.4-CWT methods to the related plant samples, the $\mathrm{DS}^{1}$ technique was subjected to the quantification of CA and RUT in the same samples. The first derivative of the absorption spectra of the calibration, validation, and plant samples were calculated with an interval of $\Delta \lambda=5 \mathrm{~nm}$ and a scaling factor of 10 in the wavelength region of $250.0-457.4 \mathrm{~nm}$. This method was named $\mathrm{DS}^{1}$ in order to save the space for the length of the paper. The first derivative spectra of the calibration samples containing CA and RUT in the working concentration range were illustrated in Figure 1d. In the case of $\mathrm{DS}^{1}$ method, the calibration curves without using the standard addition technique were obtained by measuring the first derivative amplitudes at $356.0 \mathrm{~nm}$ for CA and $390.0 \mathrm{~nm}$ for RUT (see Figure 1d). In Table 1, it was listed the statistical results of the linear regression analysis for the mentioned calibration curves of analytes. In the same way, in the method of standard addition, the calibration curves for CA and RUT were obtained by the regression analysis of the first derivative amplitudes against the concentration of analytes added to the analyzed plant sample. Based on the standard addition samples, the regression analysis results and results were indicated in Table 1. Then, CA and RUT in the analyzed plant samples were determined by using the mentioned calibration graphs of standard additions. When the slopes of calibration curves based on the regression analysis without and with standard addition were statistically compared with each other, no significant difference was observed (see Table 2). In the implementation of the CWTs and DS ${ }^{1}$ method, this showed that there was no effect of the sample matrix on the quantification of analytes in the plant samples.

Table 2. Statistical t-test results obtained from the comparison of the slopes of calibration curves without and with standard addition

\begin{tabular}{|c|c|c|c|c|c|c|}
\hline & \multicolumn{2}{|c|}{ sym8-CWT } & \multicolumn{2}{c|}{ bior4.4-CWT } & DS \\
\hline & CA & RUT & CA & RUT & CA & 356.0 \\
\hline$\lambda(\mathrm{nm})$ & 333.5 & 351.5 & 336.0 & 350.0 & -0 & -0.0369 \\
\hline $\mathrm{m}(\mathrm{I})$ & 0.1889 & 0.1205 & 0.1710 & 0.0541 & -0.0201 \\
\hline $\mathrm{m}(\mathrm{II})$ & 0.1896 & 0.1173 & 0.1692 & 0.0544 & -0.0371 & -0.0202 \\
\hline $\mathrm{t}$-calculated & 0.560 & 1.836 & 0.876 & 0.821 & 0.275 & 0.556 \\
\hline $\mathrm{t}$-crit (p=0.05) & 2.306 & 2.306 & 2.306 & 2.306 & 2.306 & 2.306 \\
\hline
\end{tabular}

$\mathrm{m}$ (I) is the slope of the regression analysis without standard addition. $\mathrm{m}$ (II) is the slope of the regression analysis with standard addition. 


\subsection{Validity of the Methods}

In the validation and implementation of the proposed sym8-CWT, bior4.4-CW methods, and $\mathrm{DS}^{1}$, good linearity with higher correlation coefficients was observed for the calibration curves of analytes as shown in Table 1. Limit of detection (LOD) and limit of quantitation (LOQ) were calculated by using the standard deviation of the linear regression equations for both CA and RUT. The values of LOD and LOQ for the calibration curves obtained by applying sym8CWT, bior4.4-CW, and $\mathrm{DS}^{1}$ approaches were calculated by the following expressions, respectively:

$$
\mathrm{LOD}=\frac{\mathrm{SD} \times 3}{\mathrm{~b}} \text { and } \quad \mathrm{LOQ}==\frac{\mathrm{SD} \times 10}{\mathrm{~b}}
$$

where SD is the standard deviation of the linear regression equation and $b$ is the slope of the linear regression equation. The statistical results of the regression analysis for both CA and RUT were listed in Table 1. In addition, the sym8CWT, bior4.4-CW, and DS ${ }^{1}$ methods based on standard addition calibrations were used for the analysis of the complex plant samples with matrix effects, because the standard addition approach has the potential power of removing these effects. In the application of all of the proposed methods, the higher correlation coefficients were reported for standard addition calibration curves as indicated in Table 1. As in the standard calibration curves, LOD, and LOQ values for the standard addition calibration curves were computed and listed in Table 1. For the proposed methods, the LOD and LOQ values were found within acceptable limits in terms of the working concentration ranges of the analytes.

In the determination of CA and RUT with sym8-CWT, bior4.4-CWT and DS ${ }^{1}$ methods, the precision and accuracy were evaluated by the analysis of the synthetic mixtures containing the analytes. Successful recoveries were obtained by application of the proposed methods to the synthetic mixture samples. These recovery results obtained by applying sym8-CWT, bior4.4-CWT and $\mathrm{DS}^{1}$ to the mixture samples were found as $99.4 \%, 99.5$, and $101.2 \%$ for CA, $98.8 \%, 102.7 \%$ and $99.1 \%$ for RUT, respectively as indicated in Table 3 . The numerical values of the standard deviation (SD) and relative standard deviation (RSD) were presented in the same table. The recovery study showed that the applied methods provided good precise and accurate results for the analysis of analytes. The low values of SD showed that the application of our proposed methods was very suitable approaches for the determination of analytes in samples.

Table 3. Recovery results for CA and RUT in their synthetic mixtures by applying CWT signal analysis and DS ${ }^{1}$ methods

\begin{tabular}{|c|c|c|c|c|c|c|c|c|c|c|c|c|c|}
\hline \multicolumn{2}{|c|}{ Added } & \multicolumn{6}{|c|}{ Found $(\mu \mathrm{g} / \mathrm{mL})$} & \multicolumn{6}{|c|}{ Recovery (\%) } \\
\hline \multicolumn{2}{|l|}{$(\mu \mathrm{g} / \mathrm{mL})$} & \multicolumn{2}{|c|}{ sym8-CWT } & \multicolumn{2}{|c|}{ bior4.4-CWT } & \multicolumn{2}{|c|}{$\mathrm{DS}^{1}$} & \multicolumn{2}{|c|}{ sym8-CWT } & \multicolumn{2}{|c|}{ bior4.4-CWT } & \multicolumn{2}{|c|}{$\mathrm{DS}^{1}$} \\
\hline $\mathrm{CA}$ & RUT & $\mathrm{CA}$ & RUT & $\mathrm{CA}$ & RUT & $\mathrm{CA}$ & RUT & $\mathrm{CA}$ & RUT & $\mathrm{CA}$ & RUT & $\mathrm{CA}$ & RUT \\
\hline 2.5 & 40 & 2.49 & 39.59 & 2.52 & 40.09 & 2.48 & 39.59 & 99.5 & 99 & 100.8 & 99.8 & 99.2 & 99 \\
\hline 5 & 35 & 4.91 & 34.18 & 5.09 & 33.78 & 5.22 & 34.18 & 98.2 & 97.7 & 101.7 & 103.6 & 104.5 & 97.7 \\
\hline 15 & 25 & 15.09 & 24.51 & 14.65 & 24.01 & 15 & 24.51 & 100.6 & 98.0 & 97.6 & 104.1 & 99.8 & 98.0 \\
\hline 25 & 15 & 24.44 & 14.58 & 24.84 & 14.58 & 25.7 & 14.58 & 97.7 & 97.2 & 99.4 & 102.9 & 102.7 & 97.2 \\
\hline 35 & 5 & 34.63 & 5.18 & 35.35 & 4.77 & 34.8 & 5.07 & 98.9 & 101.4 & 101.0 & 104.8 & 99.4 & 103.6 \\
\hline 40 & 2.5 & 40.61 & 2.48 & 38.69 & 2.48 & 40.7 & 2.48 & 101.5 & 99.2 & 96.7 & 100.8 & 101.7 & 99.2 \\
\hline & & & & & & & Mean & 99.4 & 98.8 & 99.5 & 102.7 & 101.2 & 99.1 \\
\hline & & & & & & & SD & 1.44 & 1.52 & 2.01 & 1.97 & 2.1 & 2.33 \\
\hline & & & & & & & RSD & 1.45 & 1.54 & 2.02 & 1.92 & 2.08 & 2.35 \\
\hline
\end{tabular}

$\mathrm{SD}=$ Standard deviation

RSD $=$ Relative standard deviation

To show the selectivity of the sym8-CWT and bior4.4-CWT and $\mathrm{DS}^{1}$, the standard addition samples were prepared by adding the standards of analytes (at three different concentration levels: 5.0, 20.0 and $50.0 \mu \mathrm{g} / \mathrm{mL}$ ) to the related plant sample. After applying the CWTs and DS ${ }^{1}$ methods to the standard addition samples, the added recovery results with the SD and RSD values were calculated and shown in Table 4. Analyses of the standard addition samples with the recommended signal processing methods showed that there were no interference effects of sample matrix on the determination of analytes.

Table 4. Added recovery results for CA and RUT in standard addition samples by applying CWTs and DS ${ }^{1}$

\begin{tabular}{|c|c|c|c|c|c|c|c|c|c|c|c|c|c|c|}
\hline \multirow[b]{3}{*}{ Sample } & & & \multicolumn{4}{|c|}{ sym8-CWT } & \multicolumn{4}{|c|}{ bior4.4-CWT } & \multicolumn{4}{|c|}{$\mathrm{DS}^{1}$} \\
\hline & \multicolumn{2}{|c|}{ Standard $(\mu \mathrm{g} / \mathrm{mL})$} & \multicolumn{2}{|c|}{ Found $(\mu \mathrm{g} / \mathrm{mL})$} & \multicolumn{2}{|c|}{ Recovery (\%) } & \multicolumn{2}{|c|}{ Found $(\mu \mathrm{g} / \mathrm{mL})$} & \multicolumn{2}{|c|}{ Recovery (\%) } & \multicolumn{2}{|c|}{ Found $(\mu \mathrm{g} / \mathrm{mL})$} & \multicolumn{2}{|c|}{ Recovery (\%) } \\
\hline & $\mathrm{CA}$ & RUT & $\mathrm{CA}$ & RUT & $\mathrm{CA}$ & RUT & $\mathrm{CA}$ & RUT & $\mathrm{CA}$ & RUT & $\mathrm{CA}$ & RUT & $\mathrm{CA}$ & RUT \\
\hline Plant & 5.0 & 5.0 & 5.06 & 4.82 & 101.2 & 96.3 & 4.99 & 5.08 & 99.8 & 101.6 & 5.11 & 4.93 & 102.1 & 98.5 \\
\hline Plant & 20.0 & 20.0 & 20.70 & 20.21 & 103.5 & 101.0 & 20.39 & 19.93 & 102.0 & 99.6 & 19.94 & 20.22 & 99.7 & 101.1 \\
\hline \multirow[t]{4}{*}{ Plant } & 30.0 & 30.0 & 29.94 & 29.87 & 99.8 & 99.6 & 30.76 & 31.04 & 102.5 & 103.5 & 31.36 & 28.75 & 104.5 & 95.8 \\
\hline & & & & Mean & 101.5 & 99.0 & & & 101.5 & 101.6 & & & 102.1 & 98.5 \\
\hline & & & & $\mathrm{SD}$ & 1.87 & 2.41 & & & 1.42 & 1.92 & & & 2.43 & 2.64 \\
\hline & & & & RSD & 1.84 & 2.44 & & & 1.40 & 1.89 & & & 2.38 & 2.68 \\
\hline
\end{tabular}

$\mathrm{SD}=$ Standard deviation

$\mathrm{RSD}=$ Relative standard deviation 
As described above, the standard addition method was used for the validity of the sym8-CWT and bior4.4-CWT and DS ${ }^{1}$ signal processing tools for the quantification of CA and RUT in analyzed plant samples. In addition, this standard addition method was used to reveal if there was the effect of the sample matrix's interference for the quantitative analysis of analytes in the related plant samples. In this context, the response obtained from the applied methods against the amount of standards added to the analyzed plant samples was plotted for both analytes as indicated in Figures 2a-f. Then, the analyte concentration by extrapolating the calibration curve to the $\mathrm{x}$-intercept was determined. The amount of CA and RUT obtained by applying sym8-CWT, bior4.4-CWT and $\mathrm{DS}^{1}$ methods to the standard addition samples were specified on the calibration curves of the standard addition samples as illustrated in Figure 2a-f, respectively. It was observed that the specified amount of CA and RUT in standard addition curves was in agreement with the quantitation results obtained from external standard calibration and standard addition calibration techniques to the original sample of Ribes uva-crispa. For example, when the results were multiplied by the dilution factor, the concentration of the analytes in the investigated plant sample would be found.
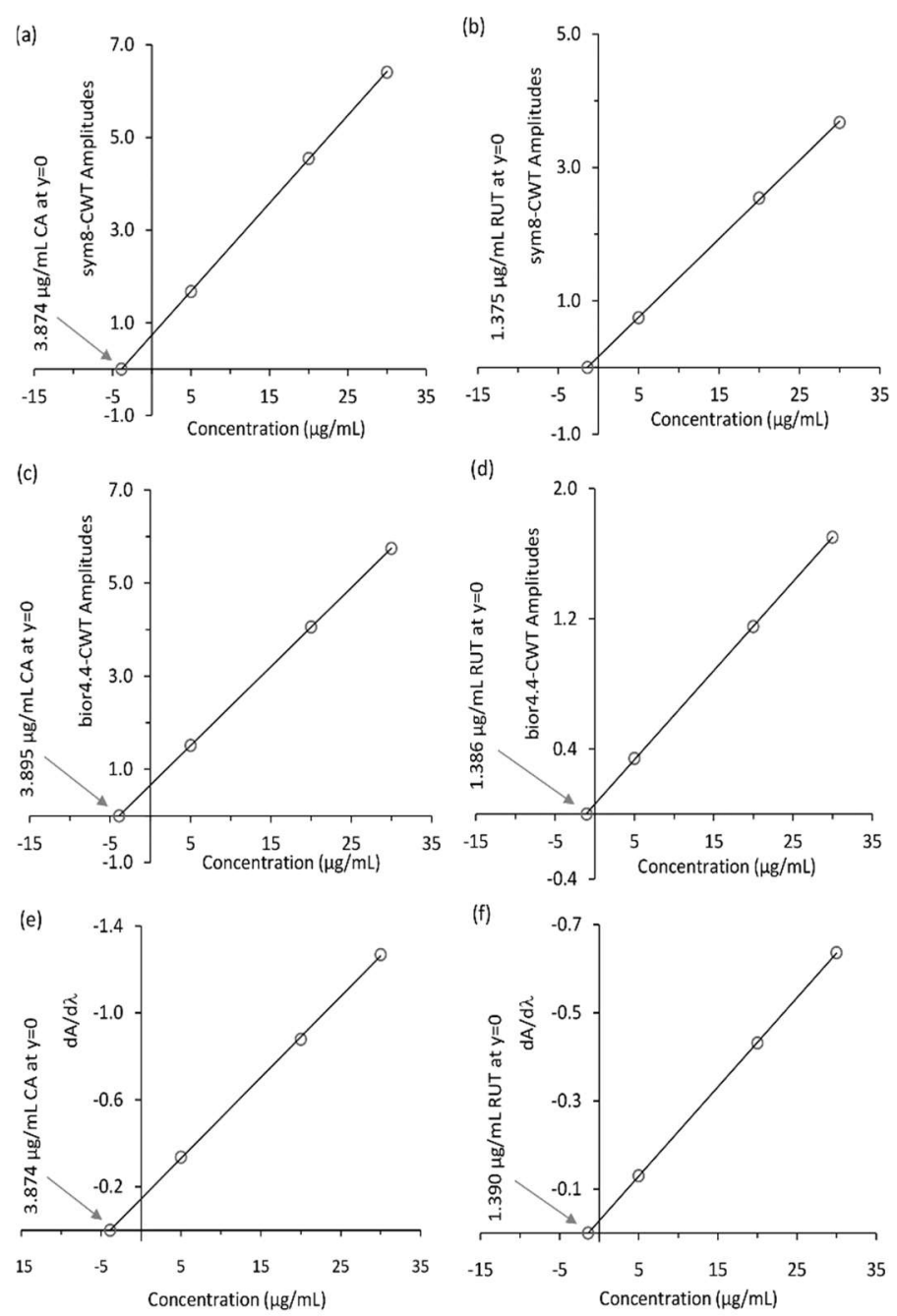

Figure 2. Plots of the CA and RUT calibration curves obtained by applying a) and b) sym8-CWT, c) and d) bior4.4-CWT, e) and f) $\mathrm{DS}^{1}$ methods, respectively, to a set of standard additions to the analyzed plant sample. The calibration curves show the CA and RUT content in analyzed plant samples when extrapolated to $\mathrm{y}=0$ was illustrated. CA : Chlorogenic acid and RUT: Rutin.

\subsection{Analysis of Plant Samples}

In the sample analysis, sym8-CWT, bior4.4-CWT and DS ${ }^{1}$ methods combined with zero-crossing technique were applied to the analysis of Ribes uva-crispa containing CA and RUT substances. The content of the analytes in leaves of $R$. uva-crispa was determined using the calibration curves without and with standard addition. The analysis results obtained were indicated in Table 5 (I) and (II). The numerical analysis values indicated that the proposed signal processing methods (sym8-CWT, bior4.4-CWT and $\mathrm{DS}^{1}$ ) were very useful for the simultaneous spectrophotometric estimation of CA and RUT in leaves of $R$. $u v a-$ crispa without using preliminary chemical separation procedure.

Table 5. Determination results of the analytes in leaves of Ribes uva-crispa by applying CWTs and DS ${ }^{1}$

\begin{tabular}{|c|c|c|c|c|c|c|c|c|c|}
\hline & & \multicolumn{8}{|c|}{$\mu \mathrm{g} / \mathrm{mg}$} \\
\hline & & \multicolumn{2}{|c|}{ sym8-CWT } & \multicolumn{2}{|c|}{ bior4.4-CWT } & \multicolumn{2}{|c|}{ DS $^{1}$} & \multicolumn{2}{|c|}{ UPLC $^{*}$} \\
\hline & & CA & RUT & $\mathrm{CA}$ & RUT & CA & RUT & $\mathbf{C A}$ & RUT \\
\hline \multirow{3}{*}{ (I) } & Mean & 21.82 & 8.56 & 21.61 & 8.37 & 21.47 & 8.52 & 22.20 & 8.50 \\
\hline & SD & 0.45 & 0.13 & 0.47 & 0.14 & 0.42 & 0.16 & 0.51 & 0.18 \\
\hline & RSD & 2.06 & 1.52 & 2.18 & 1.69 & 1.95 & 1.86 & 2.31 & 2.07 \\
\hline \multirow{3}{*}{ (II) } & Mean & 22.16 & 8.22 & 21.96 & 8.42 & 22.55 & 8.23 & - & - \\
\hline & SD & 0.47 & 0.10 & 0.25 & 0.18 & 0.55 & 0.14 & - & - \\
\hline & RSD & 2.11 & 1.21 & 1.14 & 2.18 & 2.43 & 1.72 & - & - \\
\hline
\end{tabular}

$\mathrm{SD}=$ Standard deviation

RSD $=$ Relative standard deviation .

*Analysis results in the literature [27].

(I) represents the analysis results obtained by the calibration curves without standard addition, and the experimental results were obtained from five different replications.

(II) represents the analysis results obtained by the calibration curves with standard addition, and the experimental results were obtained from three different replications.

Table 6. ANOVA tests for the analysis results of the analytes in leaves of Ribes uva-crispa by applying CWTs and DS ${ }^{1}$.

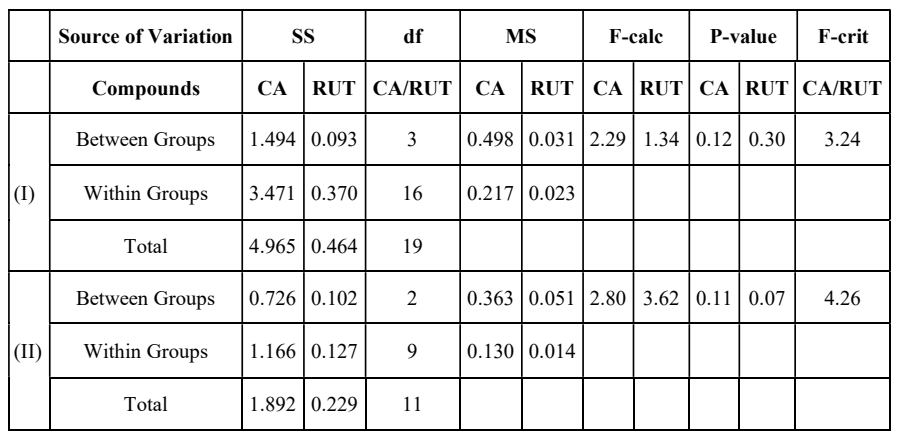

(I) is the ANOVA test for the analysis results obtained using the calibration curves without standard addition using sym8-CWT, bior4.4-CWT, DS ${ }^{1}$, and UPLC methods

(II) is the ANOVA test for the analysis results obtained using the calibration curves with standard addition using sym8-CWT, bior4.4-CWT, and DS ${ }^{1}$ methods

Considering the calibration curves of external standards, the analysis results were compared with those obtained applying the UPLC method in the literature [27]. One-way ANOVA test was applied to the analysis results provided by applying four different methods (sym8-CWT, bior4.4-CWT, DS ${ }^{1}$ and UPLC) to the investigated plant samples. The ANOVA test values at the $95 \%$ confidence level were computed and illustrated in Table 6 (I). It was observed from Table 6 (I) that the calculated F-values does not exceed the theoretical critical F-values and calculated $p$-values were greater than the theoretical p-value indicating that there is no significant difference between the accuracy of the related methods.

Similarly, the one-way ANOVA is used to compare the sym8-CWT, bior4.4CWT and DS ${ }^{1}$ determination results of analytes obtained by applying calibration curves of standard additions. In the ANOVA test, the statistical results were summarized in Table 5 (II). Results are given in Table 6 (II) showed that 
F-calculated values were lower than the theoretical critical F-values for the analysis results by the applied sym8-CWT, bior4.4-CWT and $\mathrm{DS}^{1}$ as shown in Table 6 (II). We concluded that the proposed spectral signal processing methods based on the calibration curves of standard additions were equivalent for the analysis of CA and RUT in leaves of $R$. uva-crispa.

\section{CONCLUSION}

In this research study, three signal-processing methods (sym8-CWT, bior4.4CWT and DS ${ }^{1}$ ) based on the transform of the absorption spectra were proposed for the simultaneous quantification of CA and RUT in leaves of Ribes uva-crispa. These proposed methods provided an opportunity for the precise and accurate spectral quantitation of analytes without using an UPLC analysis with low cost and a short period of analysis. In the analysis of the related analytes with sym8CWT and bior4.4-CWT and DS ${ }^{1}$, the calibration curves with and without standard addition gave comparable analysis results with the relative standard deviation between $1.21 \%$ and $2.43 \%$. In the case of the calibration curves without standard addition, sym8-CWT, bior4.4-CWT, and DS ${ }^{1}$ methods were statistically compared to the UPLC method in the literature [27]. No significant difference between experimental results was reported. Besides, the proposed three methods sym8-CWT, bior4.4-CWT, and DS ${ }^{1}$ were compared to each other. We observed that the proposed spectral methods gave the equivalent results for the analysis of CA and RUT in leaves of R. uva-crispa. As a result, this research showed that the spectral methods proposed were easy, fast, cheap, and reliable for quantitative analysis of the related medicinal compounds.

\section{FUNDING}

In this paper study, the method development and its experiment procedures were performed at the Chemometric Laboratory of Faculty of Pharmacy supported by Ankara University Scientific Research Fund (Project No. 10A3336001). This study was also supported by Ankara University Scientific Research Funds (Project numbers: 09B3336001 and 16H0237006). The authors thank Ankara University for supporting this study.

\section{REFERENCES}

1. T.C. O'Haver, T. Begley, Anal. Chem. 53, 1876-1878 (1981).

2. B. Morelli, Analyst 113, 1077-1082 (1998).

3. B. Walczak, Wavelets in Chemistry, Elsevier Press: Amsterdam, The Netherlands, 2000.

4. E. Dinç, D. Baleanu, A review on the wavelet transform applications in analytical chemistry. In Mathematical Methods in Engineering, K. Taş, J.A.T. Machado, D. Baleanu eds. Dordrecht: Springer, 2007; pp. 265- 285.

5. E. Dinç; Z. Yazan, Fron. Chem. Volume 6, Article 503, (2018).

6. I. Daubechies, Ten Lectures on Wavelets, Society for Industrial and Applied Mathematics, Philadelphia, 1992.

7. E. Dinç, FABAD J. Pharm. Sci. 38(3), 159-165 (2013).

8. E. Dinç, D. Baleanu, J. AOAC Int. 87(2), 360-365 (2004).

9. E. Dinç, D. Baleanu, J. AOAC Int. 87(4), 834-841 (2004).

10. E. Dinç, F. Arslan, D. Baleanu, J. Chil. Chem. Soc. 54(1), 28-35, (2009).

11. E. Dinç, A. Özdemir, D. Baleanu, Talanta 65, 36-47, (2005)

12. E. Dinç, D. Baleanu, Ö. Üstündağ, Spectrosc. Lett. 36, 341-355, (2003).

13. E. Dinç, A. Özdemir, D. Baleanu, J. Pharm. Biomed. Anal. 37(3), 569-575, (2005).

14. E. Dinç, D. Baleanu, Spectrochim. Acta 63(3), 631-638, (2006).

15. E. Dinç, D. Baleanu, J. Food Drug Anal. 15(2), 109-117, (2007).

16. E. Dinç, S. Kaya, T. Doğanay, D. Baleanu, J. Pharm. Biomed. Anal. 44(4), 991-995, (2007).

17. D.F. Chamberlain, Grossulariaceae. In Flora of Turkey and the East Aegean Islands, P.H. Davis ed., Edinburgh University Press: Edinburgh, Vol. 4, 1972; p. 261.

18. S. Häkkinen, M. Heinonen, S. Kärenlampi, H. Mykkänen, J. Ruuskanen, R. Törrönen, Food Res. Int. 32(5), 345-353, (1999).

19. K.R. Määttä-Riihinen, A. Kamal-Eldin, P.H. Mattila, A.M. GonzálezParamás, A.R. Törrönen, J. Agric. Food Chem. 52(14), 4477-4486 (2004).

20. M. Jordheim, F. Mage, Ø.M. Andersen, J. Agric. Food Chem. 55(14), 55295535, (2007).

21. X.L. Wu, L. Gu, R.L. Prior, S. McKay, J. Agric. Food Chem. 52(26), $7846-$ 7856, (2004).

22. M.P. Kähkönen, A.I. Hopia, M. Heinonen, J. Agric. Food Chem. 49(8), 4076-4082, (2001).

23. H. Traitler, H. Winter, U. Richli, Y. Ingenbleek, Lipids 19(12), 923-928, (1984).
24. F.D. Goffman, S. Galletti, J. Agric. Food Chem. 49(1), 349-354, (2001).

25. N. Bjarnholt, F. Rook, M.S. Motawia, C. Cornett, C. Jørgensen, C.E. Olsen, J.W. Jaroszewski, S. Bak, B.L. Møller, Phytochemistry 69(7), 1507-1516 (2008).

26. E.C. Bate-Smith, Biochem. Syst. Ecol. 4(1), 13-23, (1976).

27. G. Kendir, E. Dinç, A. Köroğlu Güvenç, J. Chromatogr. Sci. 53(9): $1577-$ $1587,(2015)$

28. E. Rácz-Kotilla, G. Rácz, Planta Med. 32(6), 110-4, (1977).

29. G. Kendir, A. Köroğlu, Int. J. Pharm. Sci. Res. 2, 108 (2015).

30. G. Kendir, A. Güvenç, S. Özkan, S. Özgen Özgacar, T. Karaoğlu, S. Gargari, Journal of Biologically Active Products from Nature 6(2), 136-149, (2016).

31. R. Duman, H.H. Doğan, M. Dinç, P. Tuncer, Int. J. Pharma Sci. Res. 9(5), 1779-1787. (2018)

32. T. Baytop, Türkiye'de Bitkiler ile Tedavi, Geçmişte ve Bugün, $2^{\text {nd }}$ ed. Nobel Tip Kitabevleri: İstanbul, 1999, p. 208

33. T.K. Lim, Edible Medicinal and Non-Medicinal Plants. Volume 4, Fruits. Springer Dordreht Heidelberg London New York, 2012.

34. L. Bunalema, S. Obakiro, J.R.S. Tabuti, P. Waako, J. Ethnopharmacol. 151(2), 999-1004, (2014)

35. F. Giusti, G. Caprioli, M. Ricciutelli, S. Vittori, G. Sagratini, Food Chem 221, 689-697, (2017)

36. M. Morvai, I. Molnar-Perl, Chromatographia 34(9/10), 502-504, (1992).

37. S. Agatonovic-Kustrin, C.M. Loescher, Anal. Chim. Acta 798, 103-108, (2013).

38. B.S. Inbaraj, H. Lu, T.H. Kao, B.H. Chen, J. Pharm. Biomed. Anal. 51(3), 549-556, (2010).

39. M.B. Hossain, D.K. Rai, N.P. Brunton, A.B. Martin-Diana, A.C. Barry-Ryan, J. Agric. Food Chem. 58(19), 10576-10581, (2010).

40. S.K.T. Seraglio, A.C. Valese, H. Daguer, G. Bergamo, M.S. Azevedo, L.V. Gonzaga, A.C.O. Costa, Food Res. Int.87, 60-67, (2016).

41. X. Wu, Y. Zhou, F. Yin, C. Mao, L. Li, B. Cai, T. Lu, Phytomedicine 21(4), $551-559,(2014)$

42. H.H.F. Koolen, F.M.A. da Silva, F.C. Gozzo, A.Q.L. de Souza, A.D.L. de Souza, Food Res. Int. 51(2), 467-473, (2013).

43. S. Kreft, M. Knapp, I. Kreft, J. Agric. Food Chem. 47(11), 4649-4652, (1999).

44. R. Pomponio, R. Gotti, M. Hudaib, V. Cavrini, J. Chromatogr. A 945(1-2), 239-247, (2002).

45. W. Wang, P. Lin, L. Ma, K. Xu, X. Lin, J. Sep. Sci. 39(7), 1357-1362, (2016).

46. A.A. Ensafi, R. Hajian, Electroanal. 18(6), 579-585, (2006). 